\title{
Death certification after a diagnosis of presenile dementia
}

\author{
A J Newens, D P Forster, D W K Kay
}

\begin{abstract}
Study Objectives-To assess the value of death certification for the epidemiological study of dementia, the frequency with which the condition was recorded on death certificates of patients diagnosed with some form of dementia before the age of 65 years was studied. A further objective was to identify variables associated with failure to record dementia on the certificate.

Design-A cohort of patients with presenile dementia, differentiated by a clinical algorithm applied to hospital case records, was traced through the National Health Service Central Registry and details of certified causes of death were obtained.
\end{abstract}

Setting-The Northern Regional Health Authority in England.

Subjects-Prevalent cases of presenile dementia resident in the northern health region during 1986 traced up to April 1992.

Measurements and main results-The underlying cause of death was recorded as dementia or as Alzheimer's disease in $\mathbf{5 3 \%}$ of cases of clinically diagnosed presenile Alzheimer's disease, $33 \%$ of cases of presenile vascular dementia, and $10 \%$ of cases of presenile dementia secondary to another neurological condition. Dementia or Alzheimer's disease was recorded in any part of the certificate in $75 \%$ of cases of Alzheimer's disease, $52 \%$ of vascular dementia, $33 \%$ of other dementias, and in $65 \%$ of cases overall. Dementia or a cerebral condition of a kind that can result in dementia was recorded in $80 \%$ of all cases. Failure to mention dementia was related to the clinical type of dementia, shorter duration of illness, and earlier period of study.

Conclusions-The underlying cause of death seriously understates the frequency of dementia, but when the recording of other brain disease is taken into account the presence of potentially dementing brain disease is recorded much more frequently. It is suggested that coding chronic conditions present at death, such as dementia, in addition to those causing or contributing to death would improve the value of death certificates for epidemiological purposes.

f Epidemiol Community Health 1993; 47: 293-297

Death certification, based on the certified underlying cause of death, has been used to map the geographical distribution of diseases. Cerebro- vascular disease and dementia have been studied in this way. ${ }^{1}$ Martyn and Pippard ${ }^{2}$ suggested that these data were not useful in exploring geographical variations in the frequency of dementia because of the low rate of the reporting of this condition in clinical cases of dementia. This has also been found in a series of well established cases of Alzheimer's disease, where dementia or Alzheimer's disease was given as the underlying cause of death in only $63 \%$ of cases ${ }^{3}$; the mean age at death in this study was 80 years.

Two large national studies ${ }^{4} 5$ have examined death certificate practices with regard to dementia amongst all deaths. In an Australian national study of regional differences in mortality from dementia that examined the underlying cause of death over a seven year period, Jorm $\mathrm{et} \mathrm{al}^{4}$ noted wide regional variations in the frequency with which dementia was reported and suggested that these were largely the result of variations in the practice of individual doctors. In a US national study ${ }^{5}$ of deaths in which senile and presenile dementia were given as the underlying causes, there was considerable underreporting of the frequency of these conditions compared with their prevalence, as estimated from epidemiological studies.

As an extension of a study that examined point prevalence, incidence, and survival in cases of presenile dementia diagnosed before the age of 65 years in the Northern Regional Health Authority, ${ }^{6}$ we report here the frequency of dementia as the underlying cause of death and compare it with the frequency of dementia as a contributory cause and with cerebrovascular disease and other cerebral diseases. Some other causes of death are also noted. We also attempt to identify variables associated with absence of dementia on the certificate, examine changes over time and differences between health districts, and study the relationship between clinically diagnosed types of dementia and causes of death given on the different parts of the death certificate. Finally, the use of death certification data for epidemiological research is discussed.

\section{Methods}

Patients with dementia or conditions likely to be associated with dementia (with the exception of Huntington's disease) were identified from the diagnostic International Classification of Diseases (ICD)-9 codes forming part of the Hospital Activity Analysis, Mental Health Enquiry, or Körner information systems from general and psychiatric hospitals. In addition, enquiries about cases of dementia were addressed to all day hospitals, social services departments, community psychiatric nurses, private nursing homes, clinical 
psychologists, and selected general practitioner practices and records of neuroradiology centres were also examined. ${ }^{6}$

Period prevalence cases of presenile dementia during the calendar year 1986 in the northern health region were identified through contact with the above services between years 1980 and 1989 inclusive. The study was restricted to patients aged between 40 and 64 years (inclusive) whose illness was diagnosed by specialist hospital services. Every case was found to have been in contact with hospital services and it was therefore possible to apply the same algorithm to case note information in order to exclude cases in whom the diagnosis of dementia was not confirmed, remove miscoded cases, and to differentiate between presenile dementia of Alzheimer's type (PDAT), presenile vascular dementia (PVD), and other secondary dementias. $^{6}$

PDAT and PVD were distinguished from one another by the clinical algorithm which took account of the type of onset and course of the disease as well as a calculation of the Hachinski ischaemia score. ${ }^{7}$ Our criteria for PVD did not require the presence of multiple infarctions and this category may have included some cases with mixed pathology. Diagnosis of secondary dementia was based solely on the clinical diagnosis as recorded in the case notes.

Patients in whom a diagnosis of dementia was confirmed by case note study were traced through the National Health Service Central Register (NHSCR) which provided a copy of the death draft entry in the register of deaths showing the underlying cause of death as reported in mortality statistics, other details of diseases contributing to death, the place of death, and whether an autopsy had been performed. ${ }^{8}$ Monitoring of death continues and deaths notified up to April 1992 are reported here.

The Office of Population Censuses and Surveys (OPCS) selects a single underlying cause of death based upon guidelines given in the ICD, modified by World Health Organisation rule three, ${ }^{9}$ and this provides the official mortality statistics. The underlying causes of death and also the immediate causes of death (cause Ia) were grouped into broad categories; Alzheimer's disease, dementia, cerebrovascular disease, cardiovascular disease, other cerebral disease, and other causes.

To take into account all the information recorded in parts I or II of the death certificate, a classification was constructed to identify separately cases with dementia, cases with central nervous system or cerebral disease, and cases without mention of either of these conditions. For this purpose clinical cases of dementia were classified as follows:

Table I Cross tabulation of clinical algorithm diagnosis of dementia and underlying cause of death

\begin{tabular}{llllllll}
\hline \multicolumn{7}{c}{$\begin{array}{l}\text { Death certificate } \\
\text { (Underlying cause of death (OPCS) }\end{array}$} \\
\cline { 2 - 8 } $\begin{array}{l}\text { Clinical } \\
\text { diagnosis }\end{array}$ & $\begin{array}{l}\text { Alzheimer's } \\
\text { disease }\end{array}$ & $\begin{array}{l}\text { Cerebro- } \\
\text { vascular }\end{array}$ & $\begin{array}{l}\text { Cardio- } \\
\text { vascular } \\
\text { disease }\end{array}$ & $\begin{array}{l}\text { Other } \\
\text { cerebral } \\
\text { disease }\end{array}$ & Other & Total \\
\hline PDAT & 32 & 59 & 27 & 28 & 4 & 23 & 173 \\
PVD & 3 & 15 & 14 & 12 & 1 & 9 & 54 \\
Other & 2 & 2 & 3 & 8 & 9 & 6 & 30 \\
Total & 37 & 76 & 44 & 48 & 14 & 38 & 257 \\
\hline
\end{tabular}

* Senile dementia, presenile dementia, and dementia

When PVD and other clinical diagnosis combined $x^{2}=22.6 \mathrm{DF}=5 \mathrm{p}<0.01$

PDAT =presenile dementia of Alzheimers type; PVD $=$ presenile vascular dementia.
(1) As Alzheimer's disease if this condition was mentioned anywhere on the certificate;

(2) As vascular dementia, if the term cerebrovascular dementia or multi-infarct dementia was recorded or if the term dementia was used in one part of the certificate and the presence of cerebrovascular disease was noted in another part;

(3) As dementia if the terms presenile dementia, senile dementia, or dementia of unspecified type were used, in the absence of Alzheimer's disease or cerebrovascular disease, (no cases had Pick's or Jacob Creutzfeldt disease recorded on the death certificate).

Cases without mention of dementia were grouped as (1) central nervous system (cerebral) diseases known to be capable of causing dementia, for example Parkinson's disease and cerebrovascular disease, or as (2) cases with no dementia or cerebral disease.

Finally, cases were allocated to one of two major groups according to whether Alzheimer's disease or dementia was or was not mentioned anywhere on the certificate, and the groups were then compared in respect of type of dementia, age at death, sex, place and year of death, and duration of illness, using the $\chi^{2}$ statistic to test for significant differences.

\section{Results}

Of 486 period prevalent cases of presenile dementia (263 men, 223 women) the NHSCR was unable to trace $35(7 \%)$, and notification of death and details of certification were available for 257 cases (141 men, 116 women). Age at death ranged from 49 to 75 years, with a mean of 64.4 years (SD $4 \cdot 4$ ) and a median age of 64.6 . A necropsy examination was carried out in $10 \%$ of these deaths. Altogether 173 cases ( 82 men, 91 women) were PDAT according to the clinical algorithm, 54 cases (38 men, 16 women) were PVD, and 30 (21 men, nine women) had other types of dementia, half of which were thought to be alcohol related. The type of dementia was significantly related to $\operatorname{sex}\left(\chi^{2}=11 \cdot 9, \mathrm{DF}=2\right.$, $\mathrm{p}<0.001)$.

\section{IMMEDIATE CAUSE OF DEATH}

(PART 1A OF CERTIFICATE)

Respiratory disease, mostly bronchopneumonia, was the commonest immediate cause of death $(48 \%)$, followed by cardiovascular disease $(16 \%)$, cerebrovascular disease $(11 \%)$, and dementia or Alzheimer's disease $(10 \%)$ There was no significant difference $\left(\chi^{2}=5 \cdot 5, \mathrm{DF}=4, \mathrm{p}>0.05\right)$ between the cause of death given in part Ia in these broad categories and PDAT or the other causes of dementia defined by the clincal algorithm.

\section{UNDERLYING CAUSE OF DEATH}

The underlying cause of death was dementia or Alzheimer's disease in $44 \%$ of cases, cerebrovascular disease in $17 \%$, and other cerebral disease in $5 \%$ (table I). Thus some kind of cerebral condition was given as the underlying cause of death in $67 \%$ of cases. Cardiovascular diseases were recorded in $19 \%$, respiratory disease in $6 \%$, and cancer in $4 \%$. Other causes constituted the remaining $5 \%$ of deaths. Of the 173 cases classed 
Table II Cross tabulation of clinical algorithm diagnosis of dementia and cause of death groups based on all death certificate entries (Ia, Ib, Ic and II)

\begin{tabular}{lllllll}
\hline \multicolumn{7}{c}{$\begin{array}{l}\text { Death certificate } \\
\text { (Cause of death group derived from all certificate entries) }\end{array}$} \\
\cline { 2 - 7 } Clinical & $\begin{array}{l}\text { Alzheimer's } \\
\text { disease }\end{array}$ & $\begin{array}{l}\text { Vascular } \\
\text { dementia }\end{array}$ & Dementia * & $\begin{array}{l}\text { Central nervous } \\
\text { system diseaset }\end{array}$ & $\begin{array}{l}\text { No dementia } \\
\text { or cerebral } \\
\text { disease }\end{array}$ & Total \\
\hline PDAT & 49 & 18 & $62 \ddagger$ & 15 & 29 & 173 \\
PVD & 5 & 11 & 12 & 12 & 14 & 54 \\
Other & 4 & 1 & $5 \S$ & 11 & 9 & 30 \\
Total & 58 & 30 & 79 & 38 & 52 & 257 \\
\hline
\end{tabular}

* Senile dementia, presenile dementia, and dementia.

TTwenty two of these neurological disorders were cerebrovascular disease.

¥Included Parkinson's disease (3) and epilepsy (1)

¿Two cases were recorded as alcohol related dementia.

as PDAT by clinical algorithm, $53 \%$ were due to Alzheimer's disease or dementia and a further $16 \%$ to a cerebrovascular condition. Of cases of PVD, 33\% were due to Alzheimer's disease or dementia and $26 \%$ to cerebrovascular disease. Of the other causes of dementia, $10 \%$ were coded as Alzheimer's disease or dementia, $10 \%$ as cerebrovascular disease, and $30 \%$ as another central nervous system disease. There was a significant difference between PDAT and all other types of dementia in the underlying cause $\left(\chi^{2}=22 \cdot 6\right.$, $\mathrm{DF}=5, \mathrm{p}<0.01)$ because of a higher percentage with Alzheimer's disease or dementia in patients with PDAT.

\section{INCLUSION OF DEMENTIA AT ANY POINT ON CERTIFICATE}

Dementia or Alzheimer's disease was mentioned somewhere on the certificate in $65 \%$ of all cases. When clinical groups were examined separately, dementia or Alzheimer's disease was mentioned in $75 \%$ of cases of PDAT, in $52 \%$ of cases of PVD, and in $33 \%$ of other dementias; this difference is highly significant $\left(\chi^{2}=24 \cdot 2, \quad D F=2, p<0.01\right)$, (table II). When any cerebral condition (including cerebrovascular disease) was included with cases of dementia, the difference between clinical groups was no longer significant (PDAT 83\%, PVD $74 \%$, and other dementia $70 \%),\left(\chi^{2}=4 \cdot 1\right.$, $\mathrm{DF}=2, \mathrm{p}>0.05$ )

When dementia was mentioned on the death certificate, this was in part II in $42 \%$ of cases and there was no significant difference between the clinical groups (table III). It should be noted that in nine cases of PDAT, Alzheimer's disease was mentioned in one section of the certificate and dementia in another and in one case of PVD arteriosclerotic dementia and dementia were both recorded.

Where Alzheimer's disease or dementia was mentioned anywhere on the death certificate, one of these conditions was recorded as the underlying cause of death in $66 \%$. Where another condition was chosen as the underlying cause, dementia or Alzheimer's disease had been recorded in part II of the certificate in $90 \%$ of these deaths.

Table III Position of dementia or Alzheimer's disease on the death certificate in relation to clinical algorithm diagnosis.
CHARACTERISTICS OF CASES WITH NO MENTION OF DEMENTIA OR ALZHEIMER'S DISEASE

\section{Diagnosis}

Altogether $35 \%$ of the certificates had no mention of dementia or Alzheimer's disease and $20 \%$ had no mention of any cerebral disease. As already noted, dementia or Alzheimer's disease was recorded significantly less often when the clinical diagnosis was PVD or other dementias than when the diagnosis was PDAT.

\section{Sex and age}

There were significantly more men than women with no mention of dementia or Alzheimer's disease $\left(x^{2}=7 \cdot 0, \mathrm{DF}=1, \mathrm{p}<0 \cdot 02\right)$, but for neither PDAT or PVD alone was the difference significant $(p>0.05)$. Younger patients (under age 65 at death) were less likely to have dementia or Alzheimer's disease mentioned than older patients $\left(\chi^{2}=4.5, D F=1, p=<0.05\right)$, a difference not related to type of dementia.

\section{Time trends}

The recording of dementia increased significantly over the study priod $\left(x^{2}=9.4, \mathrm{DF}=2, \mathrm{p}<0.02\right)$ from $54 \%$ in $1986-7$, to $68 \%$ in $1988-9$, and $76 \%$ in 1990 and later. The increase for PDAT alone did not reach a level of statistical significance $\left(x^{2}=2.9, \quad \mathrm{DF}=2, \mathrm{p}>0.05\right)$ but for PVD the increase was significant $\left(\chi^{2}=7 \cdot 5, \quad \mathrm{DF}=2\right.$, $\mathrm{p}<0.05)$. In this group, reporting of dementia or Alzheimer's disease increased from $33 \%$ in $1986-7$, to $57 \%$ in $1988-9$, and to $81 \%$ in 1990-2.

Duration of disease (onset of symptoms to death) In patients in whom the duration of disease was less than five years, dementia was reported less often $(47 \%)$ than for those with a longer disease period $(73 \%)$; this difference was highly significant $\left(\chi^{2}=15 \cdot 6, \mathrm{DF}=2, \mathrm{p}<0.01\right)$. This finding was not explained by clinical diagnosis.

Place of death

There was no significant difference in the recording of dementia or Alzheimer's disease in hospital deaths compared with those in the community. A total of 171 cases (66\%) died in hospital, $53(21 \%)$ at home and $33(13 \%)$ in a residential or nursing home. The percentage of deaths that occurred in nursing or residential homes increased during $1986-92$ (6\% in 1986-7, $9 \%$ in $1988-9$, and $16 \%$ in 1990 and later).

\section{Discussion}

The relationship between the information given on death certificates and the diagnosis during life can be regarded from two points of view. The death certificate may be used to confirm or otherwise the correctness of the clinical diagnosis, particularly when a necropsy has been performed; however, to establish the cause of dementia this would have to include histopathological examination of the brain. In this sample necropsy was undertaken in only $10 \%$ and in none was histology available.

Alternatively, death certificates may be used as a source of information about the presence of a particular disease or condition in a population; 
for instance to study regional differences in dementia. ${ }^{12}$ In addition, a reference to dementia on the certificate would suggest that the patient had been chronically disabled and dependent.

VALIDITY OF DIAGNOSIS

The general validity of the method of establishing the clinical diagnosis from case note review is supported by the calculation that in an unselected population of similar size and matched for sex and age at death, only one case of a dementing illness recorded as the underlying cause on the death certificate would be expected ${ }^{10}$ whereas in our group $113(42 \%)$ of these cases were observed. It would be wrong to assume that the remaining cases were not demented since $22 \%$ died from the effects of cerebral or cerebrovascular disease, and in a further $14 \%$ dementia or cerebral disease was mentioned on the certificate though not as the underlying cause of death. Some $80 \%$ of all patients and $83 \%$ of PDAT patients had some form of cerebral disease referred to on the certificate, which is a reasonable validation of clinical diagnosis.

There was a potential conflict between the algorithm diagnosis and the recorded causes of death in some cases. For example, of the 173 cases of PDAT cerebrovascular dementia was referred to in $18(10 \%)$ cases and in a further $19(11 \%)$ cases a cerebral disease other than Alzheimer's disease was recorded; cerebrovascular disease (11) Parkinson's disease (four), cerebral atrophy (two), epilepsy (one) cerebellar ataxia (one), (columns 3 and 4 table II). Of all 84 cases of secondary dementia (PVD and other dementias), Alzheimer's disease was mentioned on the certificate in only nine $(11 \%)$ cases (table II). It is known, however, that clinical diagnosis of type of dementia does not agree perfectly with histology. ${ }^{11} 12$

THE DEATH CERTIFICATE AS A SOURCE OF INFORMATION IN POPULATION STUDIES

The cause of death is usually reported as the underlying cause, defined under article 23 of the World Health Organisation constitution, ${ }^{13}$ as "the disease or injury which initiated the train of events leading directly to death". The ratio between the frequency with which a disease is mentioned on the death certificate and reported as the underlying cause of death varies greatly with the nature of the disease. For example, for acute myocardial infarction the ratio is 1.06 whereas for heart failure it is $19 \cdot 7 .{ }^{10}$ Therefore the underlying cause of death (on which the national mortality statistics in England and Wales are based) will accurately reflect mainly the incidence of acute conditions in which the case fatality rate is consistently high, but less so in chronic conditions. In the case of dementia, a chronic syndrome associated with varying frequency and severity with certain neurological diseases and, as age advances, with many co-morbid disorders, its contribution to death will vary and may be difficult to assess in the individual case. Hence in some cases dementia, though present, will be adjudged not to have contributed to death or else will be recorded in part II of the death certificate as contributory but unrelated to events leading to death. In this series, dementia was most often cited in this part of the certificate (table III).
Except for the ad hoc study referring to the years $1985-6,{ }^{10}$ the frequency with which dementia is noted at any point on the death certificate is not available in England and Wales. In the US, in a national study, Chandra et $\mathrm{al}^{14}$ found that age adjusted rates of dementia (senile and presenile) were about five times higher for death with any mention of these conditions than for death from these conditions when listed as the underlying cause.

In this study Alzheimer's disease or dementia was recorded was the immediate cause of death in $12 \%$ of PDAT cases (table III). This is much higher than the $1 \%$ reported as the primary (sic) cause of death in a study of older patients with Alzheimer's disease, ${ }^{3}$ and may be related to the better general health of younger patients, though the meaning of the term primary in not quite clear. As in clinical studies of older patients, ${ }^{231516}$ the commonest immediate cause of death was bronchopneumonia.

In this study Alzheimer's disease or dementia was the underlying cause of death in $44 \%$ of cases compared with $22 \%$ in the study of older patients by Martyn and Pippard. ${ }^{1}$ However, the rules for determining the underlying cause of death changed during the course of the latter study (1981-6), which may account for some of this difference. Unlike this earlier study, reporting of dementia was not related to place of death (hospital or community). Dementia or Alzheimer's disease was the commonest underlying cause of death in PDAT whereas in other clinical types of dementia death was relatively more often caused by cerebrovascular disease, other cerebral disease, or systemic disease. In just over half of cases in whom the underlying cause of death was a noncerebral cause, dementia or Alzheimer's disease was mentioned on the certificate.

In this sample, dementia or Alzheimer's disease was mentioned on any part on the death certificate in $65 \%$, about as often as reported by Martyn and Pippard $^{2}(57 \%)$, but in PDAT patients the rate was higher and similar to the $70 \%$ reported by Burns $e t a^{3}$ in older patients with Alzheimer's disease, most of whom died in hospital after a long illness. In the present study the rate of reporting in the 109 cases of PDAT who died in hospital was $79 \%$. It cannot be assumed that the failure to mention dementia in about one quarter of the cases was due to incomplete or careless certification since dementia may not have contributed to death in these cases. This is compatible with the finding that dementia was referred to less often when the duration from onset of symptoms had been less than five years than when it had been longer since dementia would have been less advanced and less likely to contribute to death from other causes.

THE NON-REPORTING OF DEMENTIA

Failure to mention dementia or Alzheimer's disease on death certificates was found in univariate analyses, to be associated with: type of dementia (PVD or other dementias as opposed to PDAT), male sex, younger age, shorter duration of illness, and earlier years of study. If cerebrovascular disease is combined with dementia as a cause of death, the difference between PDAT and PVD is reduced and the sex difference disappears. This 
procedure gives a better indication of how often the presence of cerebral disease is noted on the death certificate in the two main causes of dementia.

The increased frequency with which dementia or Alzheimer's disease are reported on the death certificates of presenile dementia cases over the past six years is perhaps the result of publicity. Although a greater proportion of patients were dying in residential homes rather than in their own home or in hospital in the later years of the study, this did not affect certification. The finding that dementia or Alzheimer's disease was mentioned more often in patients after the age of 65 than in those dying younger is somewhat surprising and may be due to chance since the difference was only of borderline significance.

\section{DEATH CERTIFICATION AND EPIDEMIOLOGICAL RESEARCH}

Martyn and Pippard ${ }^{2}$ and Jorm $e t a l^{4}$ pointed out that the use of the underlying cause of death as reported in mortality statistics would be an economical way of investigating regional differences in the incidence of dementia; unfortunately the underlying cause of death was an unreliable source of information about the presence of dementia. The coding of all conditions mentioned on death certificates and their availability for research purposes (as happens in Scotland) would improve the value of death certification. However, given the present limiting criteria for reporting conditions on the certificate, the incidence of chronic conditions like dementia would still be underestimated. The addition of a section for chronic conditions present but not necessarily contributing to death would improve the value of death certificates as a source of information about the frequency of disabling conditions such as dementia likely to have caused dependency before death. Such a proposal would probably need international agreement but nevertheless is one that justifies serious consideration, particularly at a time when health need assessment of chronic conditions assumes such importance in purchasing health care.

We are very grateful to the Northern Regional Health Authority and the Medical Research Council for funding; to Mr Angus McNay and the statistics section of the NRHA and to the NHSCR at Southport for help with information.

1 Gardner MJ, Winter PD, Barker DJP. Atlas of mortality from selected diseases in England and Wales 1968-78. Chichester John Wiley, 1984: 62-3.

2 Martyn CN, Pippard EC. Usefulness of mortality data in determining the geography and time trends of dementia. $f$ Epidemiol Community Health 1988; 42: 134-7.

3 Burns A, Jacoby R, Luthert P, Levy R. Cause of death in Alzheimer's disease. Age Ageing 1990; 19: 341-4.

4 Jorm AF, Henderson AS, Jacomb PA. Regional differences in mortality from dementia in Australia: an analysis of death certificate data. Acta Psychiatrica Scandanavia 1989; 79; 179-85.

5 Jordan BD, Schoenberg BS. Mortality from senile dementi in the United States. South Med $\mathcal{F}$ 1986; 76: 5, 529-31

6 Newens AJ, Forster DP, Kay DWK, Kirkup W, Bates D Edwardson J. Clinically diagnosed presenile dementia of Alzheimer type in the northern health region: ascertainment, prevalence, incidence, and survival. Psychol Med 1993; 23: (in press).

7 Hachinski, VC, Iliff LD, Zilhka E, et al Cerebral blood flow in dementia. Arch Neurol 1975; 32: 632-7.

8 Fox AJ. The work of the national health service centra register. Population Trends 1990; 62: 29-32.

9 Office of Population Censuses and Surveys Mortality statistics 1984: cause. London: HMSO, 1985. Series DH2, no 11.

10 Office of Population Censuses and Surveys Mortality statistics 1986: cause. London: HMSO, 1988. Series DH2, no 13.

11 Mölsa PK, Marttila RJ, Rinne UK. Survival and cause death in Alzheimer's disease and multi infarct dementia. Acta Neurol Scand 1986; 74; 103-7.

12 Joachim CL, Morris JH, Selkoe DJ. Clinically diagnosed Alzheimer's disease: autopsy results in 150 cases. Ann Neurol 1988; 24: 50-56.

13 World Health Organisation. Official record. Geneva: World Health Organisation, 1950.

14 Chandra V, Bharucha NE, Schoenberg BS. Patterns of mortality from types of dementia in the United States, 1971 and 1973-1978. Neurology 1986; 36: 204-8.

15 Kay DWK. Outcome and cause of death in mental disorders of old age. Acta Psychiatrica Scandinavia 1962; 38: 249-58

16 Peck A, Wolloch L, Rodstein M. Mortality of the aged with chronic brain Syndrome: further observations in a five year study. F Am Geriatr Soc 1978; 28: 170-6. 